\title{
Volatile Sulfur Production in the Large Intestine as Affected by Plant Extracts in Pig In Vitro Model
}

\author{
Yoshimi Ishikawa, ${ }^{1}$ Makiko Maekawa, ${ }^{1}$ Tsutomu Arakawa ${ }^{2}$ and Kazunari Ushida ${ }^{1 *}$ \\ 'Laboratory of Animal Science, Kyoto Prefectural University, Shimogamo, Sakyo-ku, Kyoto 606-8522, Japan \\ ${ }^{2}$ LOTTE Central Laboratory, Numakage, Urawa, Saitama 336-0027, Japan \\ Received October 16, 2000; Accepted for publication, April 9, 2001
}

\begin{abstract}
Extracts of several Oriental and European herbs were tested for their inhibitory effects on volatile sulfur production in pig in vitro cecal fermentation. Some of the extracts, namely, Marrubium vulgare and Perilla frutescens, significantly decreased volatile sulfur and ammonia production. The present results indicate that some herb extracts might improve fecal malodor and possibly host health.
\end{abstract}

Key words: hydrogen sulfide; methanethiol; large intestine; pig; European and Oriental herbs

Volatile sulfur compounds (VS), such as hydrogen sulfide, methanethiol, and dimethyl sulfide, are generated in the large intestine by bacterial metabolism. The bacteria concerned with VS production have a capacity to reduce free sulfate and/or desulfhydrate sulfur amino acids by $\alpha$ - or $\beta$ - $\gamma$ elimination. The former is categorized as sulfate-reducing bacteria such as Desulfovibrio spp., and some species of clostridia and fusobacteria are involved in the latter $(7,13)$. Hydrogen sulfide injures colonocytes (17) and might be a pathogenesis of ulcerative colitis in human subjects $(4$, 16). Aminoglycoside antibiotic inhibits luminal sulfide production by sulfate-reducing bacteria in ulcerative colitis, suggesting the significant contribution of sulfate-reducing bacteria in this disease (14). Removing foods rich in sulfur amino acids, such as milk, egg, and cheese, has improved ulcerative colitis (17), suggesting a similar importance of sulfur amino acidsdesulfhydrating bacteria in this desease. Methanethiol generated from L-methionine by intestinal bacteria may cause hepatic encephalopathy in rats, dogs, and cirrhotic patients $(3,11)$. The control of VS production has therefore a clinical importance, particularly in the abovementioned patients. Otherwise these compounds are the cause of fecal malodor. The reduction of fecal odor has practical values not only in the care of human but also in integrated animal production. Jantea et al. (6) indicated that sulfide and indole-producing bacteria increased in human hindgut as age increased. Dietary supplements that decrease the production of these compounds in the large intestine and in excretion in feces of aged subjects will be valuable for human care. For these purposes, we have previously selected natural

${ }^{*}$ Corresponding author. Mailing address: Laboratory of Animal Science, Kyoto Prefectural University, Shimogamo, Sakyo-ku, Kyoto 606-8522, Japan. Phone/Fax: +81-75-703-5620. E-mail: k_ushida@kpu.ac.jp products, namely European and Oriental herb extracts that reduced the hydrogen sulfide production of sulfate-reducing bacteria ( 1 ). In this experiment, we tried to further determine the inhibitory effects of various herb extracts on VS production in the large intestine with pig in vitro model. Pig large intestine is considered a potential model for human large intestine in certain aspects, such as fiber and starch digestion and flora composition $(5,10,12,21)$.

Herb extracts were prepared from the following 11 herbs: Marrubium vulgare (white horehound), Hyssopus officinalis (hyssop), Ajuga decumbens (Kiranso), Alliium schoenoprasum (chives), Mentha piperita (peppermint), Glechoma hederacea (Kakidoshi), Curcuma longa (turmeric), Artemisia princeps (Yomogi), Ocimum basilicum (basil), Perilla frutescens (Soyo, or perilla), and Myrtus communis (myrtle). The herbs were purchased from UchidaWakanyaku (Tokyo, Japan) and KARIS-Seijo (Tokyo, Japan) and extracted as indicated previously (1). Most extracts were prepared as aqueous extracts except for $O$. basilicum, $P$. frutescens, and $M$. communis. The latter two were extracted with 50\% aqueous ethanol, and the former was extracted with ethanol. The products were then freeze-dried. Two mature female crossbred pigs (Landrace $\times$ Large white $\times$ Duroc) about $120 \mathrm{~kg}$ B.W. were used as donors of cecal fluid. They were fistulated at the cecum to enable cecal digesta sampling. The pigs were fed a meal composed of alfalfa meal ( 250 $\mathrm{g})$, meat bone meal ( $250 \mathrm{~g})$, and cracked maize $(820 \mathrm{~g})$. The daily diet was supplemented with $\mathrm{NaCl}, \mathrm{CaCO}_{3}$, and vitamin-mineral premix (Kokin Kagaku, Osaka, Japan) according to the Japanese Feed Standard for pigs (20). Water and mineral block (Green Kouen, Nihon Zenyaku, Fukushima, Japan) were always available for the animals. The dietary component was obtained from 
Table 1. Effect of herb extracts on volatile sulfur production $\left(10^{-3} \mathrm{ppm}\right.$ in headspace $)$ in pig cecal digesta in vitro.

\begin{tabular}{lccccc}
\hline \multirow{2}{*}{ Herb extract } & \multicolumn{2}{c}{ Hydrogen sulfide produced from } & \multicolumn{2}{c}{$\begin{array}{c}\text { Methanethiol } \\
\text { produced from }\end{array}$} \\
\cline { 2 - 6 } & None & Sodium sulfate & L-Cystine & L-Methionine & L-Methionine \\
\cline { 2 - 6 } & $1.20 \pm 0.02$ & $15.32 \pm 1.49$ & $72.11 \pm 9.74$ & $2.12 \pm 0.22$ & $14.93 \pm 2.17$ \\
None & $1.43 \pm 0.24$ & $9.02 \pm 1.56^{*}$ & $49.92 \pm 7.83^{*}$ & $2.45 \pm 0.84$ & $14.51 \pm 6.08$ \\
Marrubium vulgare & $1.55 \pm 0.63$ & $9.11 \pm 1.96^{*}$ & $59.93 \pm 1.24$ & $2.08 \pm 0.28$ & $12.07 \pm 1.52$ \\
Hyssopus officinalis & $1.00 \pm 0.04$ & $13.29 \pm 3.06$ & $55.21 \pm 3.32$ & $1.94 \pm 0.51$ & $10.77 \pm 1.03$ \\
Ajuga decumbens & $2.15 \pm 1.89$ & $12.70 \pm 3.55$ & $49.85 \pm 1.46^{*}$ & $1.54 \pm 0.73$ & $11.02 \pm 1.27$ \\
Alliium schoenoprasum & $1.92 \pm 0.59$ & $10.47 \pm 1.10^{*}$ & $76.12 \pm 6.33$ & $1.50 \pm 0.07$ & $10.94 \pm 0.42$ \\
Mentha piperita & $1.32 \pm 0.34$ & $13.53 \pm 2.12$ & $62.15 \pm 7.35$ & $1.13 \pm 0.03 *$ & $10.18 \pm 1.82$ \\
Glechoma hederacea & $2.13 \pm 0.83$ & $6.45 \pm 2.31^{*}$ & $77.65 \pm 0.34$ & $2.00 \pm 0.05$ & $15.22 \pm 2.93$ \\
Curcuma longa & $1.28 \pm 0.52$ & $13.64 \pm 2.64$ & $86.12 \pm 3.08$ & $2.49 \pm 0.34$ & $12.44 \pm 9.07$ \\
Artemisia princeps & $1.13 \pm 0.33$ & $12.72 \pm 3.32$ & $61.61 \pm 4.71$ & $1.98 \pm 0.01$ & $10.18 \pm 2.46$ \\
Ocimum basilicum & $0.96 \pm 0.64$ & $6.66 \pm 1.63^{*}$ & $86.93 \pm 6.84$ & $1.08 \pm 0.50$ & $8.62 \pm 1.31^{*}$ \\
Perilla frutescens & $0.72 \pm 0.54$ & $8.85 \pm 0.71 *$ & $69.33 \pm 7.40$ & $1.55 \pm 0.01$ & $11.75 \pm 1.54$ \\
Myrtus communis & &
\end{tabular}

Culture was done in diluted cecal contents with MES buffer $(50 \mathrm{~mm}, \mathrm{pH}$ 6.5) for $24 \mathrm{hr}$ under anaerobic conditions. Cultures were amended with sodium sulfate $(10 \mathrm{mM})$, L-cystine $(1 \%, \mathrm{w} / \mathrm{v})$, or $\mathrm{L}$-methionine $(1 \%, \mathrm{w} / \mathrm{v})$. Herb extracts were added to the culture at $0.1 \mathrm{~g} / \mathrm{l}$. Values are means with standard deviations for four determinations. Values with asterisks were significantly different $(p<0.05)$ from those without herb extracts (none).

Kumiai Shiryo (Kobe, Japan) unless otherwise stated. Cecal digesta was sampled $1 \mathrm{hr}$ after morning feeding from two pigs and combined. The digesta were further diluted with a volume of 2-morphorinoethane sulfonic acid ( $50 \mathrm{~mm}, \mathrm{pH} 6.5$ ). The buffer was boiled and cooled under $\mathrm{N}_{2}$ before use. The mixture was homogenized and squeezed through two layers of surgical gauze un$\operatorname{der} \mathrm{N}_{2}$ atmosphere. A portion $(5 \mathrm{ml})$ of filtrate was introduced in a $10 \mathrm{ml}$ serum vial and closed with a butyl rubber septum (Bellco Laboratories, Vineland, NJ, USA). L-Methionine $(1 \%, \mathrm{w} / \mathrm{v})$, L-cystine $(1 \%, \mathrm{w} / \mathrm{v})$, or sodium sulfate $(10 \mathrm{~mm})$ was added to the culture as a substrate or an electron acceptor to induce VS production by particular pathways; this might help to specify the target of herb extracts when inhibitory effects were seen. Cultures without these substrates or an electron acceptor were also done. Eleven herb extracts as shown in Table 1 were added to the culture at the final concentration of $0.1 \mathrm{~g} / l$. Freeze-dried extracts were dissolved in distilled water or ethanol and poured into vials before an addition of the diluted digesta. For the ethanol-dissolved extracts, ethanol was evaporated from vials with $\mathrm{N}_{2}$ gas stream before an addition of digesta and substrates. The concentration of herb extracts was selected according to the preliminary experiment where selection was made, based on the inhibitory effect on sulfide production by sulfate-reducing bacteria at a dose level of $0.1 \mathrm{~g} / l(1)$. Vials were further closed with aluminum crimps and allowed to stand for $24 \mathrm{hr}$ at $37^{\circ} \mathrm{C}$. At the end of incubation, $6 \mathrm{~N} \mathrm{HCl}(1 \mathrm{ml})$ was added to the culture to quickly stop fermentation and release soluble VS into headspaces. A portion $(0.5 \mathrm{ml})$ of headspace gas was sampled by a gas-tight syringe and analyzed for VS by gaschromatography as indicated elsewhere $(1,22)$. A portion of culture fluids was also analyzed for short-chain fatty acid concentration by ionexclusion HPLC (23). Four vials were used for each treatment. Values were treated with Student's $t$-test to detect statistical significance with and without herb extracts in each culture condition.

VS was detected in all conditions; Hydrogen sulfide was detected at over 1,000 ppm even in cultures without substrate $(=$ None). Substrate for VS production such as sodium sulfate, L-cystine, and L-methionine stimulated hydrogen sulfide and methanethiol production. Hydrogen sulfide was produced 10 and 50 times more in sulfate and L-cystine amended cultures, respectively, than cultures without substrate (Table 1). L-Methionine amendment increased hydrogen sulfide production only 1.75 times, but methanethiol production was detected at high concentration only in the L-methionine-amended culture (about 15,000 ppm). The concentration was small (less than $10 \mathrm{ppm}$ ) in the other culture conditions. Therefore methanethiol production was discussed only in the L-methionine-amended cultures. Dimethyl sulfide was detected in very small amounts even in the L-methionine-amended cultures. Therefore this VS will not be further discussed.

Without substrate for VS production (Table 1), there was little difference between cultures with and without herb extracts in hydrogen sulfide production. In sulfate-amended cultures, the extracts of $M$. vulgare, $H$. 
Table 2. Effect of herb extracts on ammonia production ( $\mathrm{mg} \mathrm{N} / \mathrm{dl}$ increased) in pig cecal digesta in vitro.

\begin{tabular}{lcccc}
\hline \multirow{2}{*}{ Herb extract } & \multicolumn{3}{c}{ Sulfur sources } \\
\cline { 2 - 4 } & None & Sodium sulfate & L-Cystine & L-Methionine \\
\hline None & $14.1 \pm 0.1$ & $17.1 \pm 1.9$ & $45.5 \pm 2.9$ & $30.0 \pm 3.6$ \\
Marrubium vulgare & $12.4 \pm 1.2$ & $13.7 \pm 0.7$ & $43.7 \pm 4.5^{*}$ & $25.0 \pm 2.2$ \\
Hyssopus officinalis & $12.7 \pm 0.9$ & $14.0 \pm 0.1$ & $38.9 \pm 1.8^{*}$ & $23.8 \pm 0.7^{*}$ \\
Ajuga decumbens & $13.6 \pm 0.4$ & $13.8 \pm 1.0$ & $40.8 \pm 1.6^{*}$ & $21.9 \pm 0.4^{*}$ \\
Alliium schoenoprasum & $10.1 \pm 5.2$ & $15.3 \pm 0.4$ & $42.8 \pm 3.5$ & $21.4 \pm 0.7^{*}$ \\
Mentha piperita & $12.8 \pm 0.9$ & $14.4 \pm 0.9$ & $41.1 \pm 3.1^{*}$ & $20.5 \pm 0.1^{*}$ \\
Glechoma hederacea & $13.5 \pm 0.1$ & $14.2 \pm 1.2$ & $41.0 \pm 3.0^{*}$ & $24.9 \pm 1.0^{*}$ \\
Curcuma longa & $15.9 \pm 0.0$ & $13.9 \pm 0.1 *$ & $37.4 \pm 1.1^{* *}$ & $25.0 \pm 0.5^{*}$ \\
Artemisia princeps & $12.5 \pm 0.5$ & $14.1 \pm 0.6$ & $35.9 \pm 1.1^{* *}$ & $25.1 \pm 0.5$ \\
Ocimum basilicum & $14.9 \pm 1.1$ & $14.4 \pm 0.7$ & $42.2 \pm 1.8^{*}$ & $26.7 \pm 5.8$ \\
Perilla frutescens & $12.2 \pm 0.3 *$ & $15.0 \pm 0.5$ & $48.8 \pm 0.0$ & $25.8 \pm 0.4^{*}$ \\
Myrtus communis & $11.2 \pm 1.0^{*}$ & $14.2 \pm 0.5$ & $43.5 \pm 0.4^{*}$ & $26.3 \pm 0.1^{*}$ \\
\hline
\end{tabular}

Culture conditions, see Table 1. Values with asterisks were significantly different $\left({ }^{*} p<0.05,{ }^{* *} p<0.01\right)$ from those without herb extracts (none).

officinalis, M. piperita, C. longa, $P$. frutescens, and $M$. communis significantly decreased hydrogen sulfide production. When L-cystine was supplemented to stimulate hydrogen sulfide production by $\beta$-elimination reaction, the extracts of $M$. vulgare and $A$. schoenoprasum notably decreased hydrogen sulfide production. In the L-methionine amended cultures, methanethiol was produced by desulfhydration of L-methionine by $\alpha-\gamma$ elimination. The effect of $P$. frutescens extract was significant because $40 \%$ of methanethiol production was reduced in those cultures. The herb extracts were mostly inhibitory against VS production in sulfate-amended cultures. No herb extracts, however, showed significant effects on VS productions throughout the culture conditions used. Only two, such as $M$. vulgare and $P$. frutescens, showed significant effects on VS production under two different culture conditions. This incompatibility in the inhibitory effect of herb extracts may partly be due to the dose level of herb extracts. In the first screening experiment (1), we adopted three dose levels of extracts $(0.01,0.1$, and $1.0 \mathrm{~g} / l)$. None of the extracts showed any inhibitory effect on hydrogen sulfide production in pure cultures of Desulfovibrio desulfuricans DSM 642 at the lowest level, and all the extracts significantly reduced it at the highest level. These two dose levels were inappropriate for the screening purpose. Therefore we adopted a middle level of dose $(0.1 \mathrm{~g} / l)$ for the second screening step. However, this dose level might be insufficient for most herb extracts to affect the complex bacterial system such as diluted cecal fluid. The incompatibility in results also suggested the different antibacterial spectra of inhibitory substances involved. In the sulfate-amended cultures, sulfate-reducing bac- teria were the principal organisms producing hydrogen sulfide, thus the principal target of herb extracts. As indicated above, it seems logical that most of the tested herb extracts were inhibitory against VS production in sulfate-amended cultures because they had been selected from the first screening step by the effect on sulfate reduction by $D$. desulfuricans $(1)$. In the L-cystineamended cultures, the bacteria that have L-cysteine $\beta$ lyase activity were the principal target. According to Persson et al. (13), several species in the genera Peptostreptococcus, Eubacterium, Bacteroides, Fusobacterium, and Selenomonas have high potential to produce hydrogen sulfide from L-cysteine. Probably these bacteria were responsible for hydrogen sulfide production from L-cystine in the present culture condition, because these are the common colonizers in pig large intestine $(15,18)$. L-Cystine must be reduced to L-cysteine by cystine reductase before degradation. This reaction occurs rapidly, and the resultant L-cysteine is further desulfhydrolyzed in the rumen of sheep to produce hydrogen sulfide (2). Judged from the similarity between the sheep rumen and the pig cecum, namely anaerobiosis and similarity of the major flora constitution, this also occurred in the present cultures. In the $\mathrm{L}-$ methionine-amended cultures, the bacteria having Lmethionine $\alpha-\gamma$ lyase were the principal target of herb extracts. Therefore some species of genera Clostridium, Fusobacterium, Porphyromonas, Eubacterium, and Bacteroides $(7,13,19)$ were the most probable target in these cultures. The latter two bacteria are the predominant species in pig large intestine $(15,18)$. The bacteria belonging to the former two genera are not the major colonizers of the pig large intestine, but they still 
constitute the hindgut flora in a substantial number, $1 \%$ to $2 \%$ of total isolates $(15,18)$.

The ammonia concentrations were higher in L-methionine- and L-cystine-amended cultures, suggesting that the deamination occurred at the same time of the desulfhydration of these sulfur amino acids. Ammonia is a major compound in the hindgut and is often considered tumorigenetic (8). Most herb extracts tested slightly, but significantly decreased the ammonia production (Table 2). Amino acid deamination in the rumen was notably inhibited by plant essential oils (10). At present the major components in the presently used herb extracts are unknown. In our preliminary experiments, our herb extracts were subjected to a serial organic solvent extraction. Hexane, ethyl acetate, and $n$ butanol fractions had a strong inhibitory effect on VS production (data not shown). This probably suggests that not only essential oils, but also flavonoids and saponins must be involved in the effective components. This requires further elucidation.

The present results indicate that two plant extracts, $M$. vulgare and $P$. frutescens, merit further testing in in vivo studies.

\section{REFERENCES}

(1) Arakawa T, Ishikawa Y, Ushida K. 2000. Volatile sulfur production by pig cecal bacteria in batch culture and screening inhibitors of sulfate-reducing bacteria. J Nutr Sci Vitaminol 46: 159-164.

(2) Bird PR. 1972. Sulphur metabolism and excretion studies in ruminants. IX. Sulphur, nitrogen, and energy utilization by sheep fed a sulphur deficient and a sulphate-supplemented, roughage-based diet. Aust J Biol Sci 25: 10731085.

(3) Blom HJ, Chamuleau RA, Rothuizen J, Deutz NE, Tangerman A. 1990. Methanethiol metabolism and its role in the pathogenesis of hepatic encephalopathy in rats and dogs. Hepatology 11: 682-689.

(4) Florin THJ, Gibson GR, Neale JH, Cummings JH. 1990. A role for sulfate for reducing bacteria in ulcerative colitis? Gastroenterology 98: A170.

(5) Graham H, Aman P. 1986. The pigs as a model in dietary fiber digestion. Scan J Gastroenterol 22: 55-61.

(6) Jantea F, Nicolae D, Bad-Oprisescu D, Voina P. 1965. Beitrag zur darmmikroflora bei menschen im alter von 45100 jahren. Ernaehrungsforschung 10: 352-362.

(7) Kreis W, Hession C. 1973. Isolation and purification of $L_{-}-$ methionine- $\alpha$-deamino- $\gamma$-mercaptomethane-lyase ( $\mathrm{L}$ - methioninase) from Clostridium sporogenes. Cancer Res 33: $1862-1865$.

(8) Lin HC, Visek WJ. 1991. Large intestinal pH and ammonia in rats: dietary fat and protein interactions. J Nutr 121: 832-843.

(9) McBurney MI, Saner WC. 1993. Fiber and largebowel energy absorption: Validation of the integrated ileostomy fermentation model using pigs. J Nutr 123: 721-727.

(10) McIntosh FM, Newbold CJ, Losa R, Williams P, Wallace RJ. 2000. Effects of essential oils on rumen fermentation. Reprod Nutr Dev 40: 221-222.

(11) Merino GE, Jetzer T, Doizaki WM, Najarian JS. 1975. Methionine-induced hepatic coma in dogs. Am J Surg 130: 41-46.

(12) Mitsuoka T, Kaneuchi C. 1977. Ecology of the bifidobacteria. Am J Clin Nutr 30: 1799-1810.

(13) Persson S, Edlund MB, Claesson R, Carlsson J. 1990. The formation of hydrogen sulfide and methyl mercaptan by oral bacteria. Oral Microbiol Immunol 5: 195-201.

(14) Pitcher MCL, Beaty ER, Gibson GR, Cummings JH. 1995. Incidence and activities of sulphate reducing bacteria in patients with ulcerative colitis. Gut 36: A63.

(15) Robinson IM, Allison MJ, Bucklin JA. 1981. Characterization of the cecal bacteria of normal pigs. Appl Environ Microbiol 41: 950-955.

(16) Roediger WE, Duncan A, Kapaniris O, Millard S. 1993. Reducing sulfur compounds of the colon impair colonocyte nutrition: implications for ulcerative colitis. Gastroenterology 104: 802-809.

(17) Roediger WEW, Moore J, Babidge W. 1997. Colonic sulfide in pathogenesis and treatment of ulcerative colitis. Dig Dis Sci 42: 1571-1579.

(18) Russell EG. 1979. Types and distribution of anaerobic bacteria in the large intestine of pigs. Appl Environ Microbiol 37: 187-193.

(19) Tang-Larsen J, Claesson R, Edlund MB, Carsson J. 1995. Competition for peptides and amino acids among periodontal bacteria. J Periodontal Res 30: 390-395.

(20) The Ministry of Agriculture, Forestry and Fisheries. 1998. Japanese Feeding Standard for Swine.

(21) Topping DL, Gooden JM, Brown IL, Biebrick DA, McGrath L, Trimble RP, Choct M, Illman R. 1997. A high amylose (Amylomaize) starch raises proximal large bowel starch and increase colon length in pigs. J Nutr 127: 615 622.

(22) Ushida K, Fukusada S, Kojima Y. 1998. Effect of pH on dissimilatory sulfate reduction by porcine cecal microflora. Anim Sci Technol (Jpn) 69: 571-575.

(23) Ushida K, Sakata T. 1998. Effect of pH on oligosaccharide fermentation by porcine cecal digesta. Anim Sci Technol (Jpn) 69: 100-107. 